\title{
A Murine Effort Model for Studying the Influence of Trichinella on Muscular Activity of Mice
}

\author{
Ionuț MARIAN*, Călin-Mircea GHERMAN, Andrei Daniel MIHALCA \\ University of Agricultural Sciences and Veterinary Medicine Cluj-Napoca, Department of Parasitology and Parasitic Diseases, Calea Mănăștur 3-5, Cluj-Napoca, \\ 400372,Romania; klis87ionut@yahoo.com ("correspondingauthor); calin.gherman@usamvcluj.ro; amihalca@usamvcluj.ro
}

\begin{abstract}
Trichinella are nematodes parasitic in the skeletal muscles of terrestrial vertebrates, generally transmitted via predatorism. It is expected that the infection would have certain influences on the muscular activity in infected animals. The aim of the study was to develop an experimental model for studying the muscular effort in laboratory mice prior to the experimental infection with Trichinella and to evaluate the method in trained (with free access to a voluntary activity wheel) and untrained (without access to activity wheel) animals. Ten laboratory mice (all adult males), equally divided in two groups were used: a control group (untrained mice) and a second group (trained). The weight was evaluated individually. The muscular activity was evaluated using an effort-wheel. Values were expressed in instantaneous power (IP) and data were recorded using a constant speed of $5 \mathrm{rpm}$ for 5 and 20 minutes. The instantaneous power (IP) developed by the effort wheel at 5 minutes was significantly lower in the control group than in trained mice. Similar results were obtained for the maximum power (MP). Interestingly, for the trained mice, there was no difference between the average IP at 5 and 20 minutes of activity. The results show the utility of trained mice, establish the necessary experiment time and validate the method for evaluating the influence of Trichinella spp. on the muscular activity of experimentally infected mice.
\end{abstract}

Keywords: experimental model, muscular activity, effort wheel, Trichinella

\section{Introduction}

Nematodes of genus Trichinella are parasitic in the skeletal muscles of terrestrial vertebrates. In humans, the infection is severe, with significant mortality in untreated patients. However, the natural infection in animals is usually asymptomatic (Despommier, 1983). Nevertheless, as in natural cycles, Trichinella spp. are transmitted via predatorism, and thus certain influences of the infection on the muscular activity in infected animals can be expected.

There are many known situations when the trophic transmission of parasites is associated with detrimental effect on the prey host and increased chance of predation by carnivorous hosts (Moore, 2002). Such examples include various parasitic transmission models, some experimental and some of them under natural conditions. Most of these situations are defined as "behavioral alterations" (Moore, 2002) as the examinations were purely observational, without a clear demonstration of the associated morpho-functional changes.

However, despite the multitude of parasites known to alter the behavior of the prey hosts, surprisingly, the effect of Trichinella in this regard still remains uninvestigated using nonvoluntary effort as an imitation of natural predator escape strategy. As Trichinella is parasitic in the skeletal muscles of the prey host and its transmission to the next host is via predatorism, the hypothetical expected effect is on the muscular performance.
There are several ways to investigate this in laboratory animals, but the two main models used are the effort wheel (induced activity) and activity wheel (voluntary activity) (Novak et al., 2012).

The aim of the study was to develop an experimental model for studying the efficacy of induced muscular effort in laboratory mice prior to the experimental infection with Trichinella and to evaluate the efficacy of the protocol in trained and untrained animals.

\section{Materials and Methods}

There were used 10 laboratory mice, divided in two groups: a control group of untrained mice $(n=5)$ and a second group of trained mice $(n=5)$. The training of mice in the second group was achieved by keeping them in individual cages with free access to a voluntary activity wheel (IntellioBio) for three weeks prior to the experiment. The mice in the control group where housed individually in cages without access to activity wheel. Each group consisted of adult males. The weight of the mice was evaluated individually prior to the experimental trials.

In order to evaluate the muscular performance, two experimental designs were designed. In the first trial, the muscular effort capacity was compared between the mice in the control group and the trained mice, over a duration of 5 minutes. In the second trial (performed 24 
270

Table 1. Data for the trained group at effort activity for 5 minutes

\begin{tabular}{ccccc}
\hline Mouse & Status & RPM & Power average (IP) & Power max (MP) \\
\hline 1 & Trained & 5.24 & 211.38 & 370.88 \\
2 & Trained & 5.29 & 221.55 & 410.90 \\
3 & Trained & 5.16 & 177.68 & 289.19 \\
4 & Trained & 5.20 & 182.71 & 289.37 \\
5 & Trained & 5.26 & 214.35 & 373.69 \\
& Mean $($ std) & & $201.53 \pm 19.91$ & $346.80 \pm 54.84$ \\
\hline
\end{tabular}

Table 2. Data for the untrained (control) group at effort activity for 5 minutes

\begin{tabular}{ccccc}
\hline Mouse & Status & RPM & Power average (IP) & Power max (MP) \\
\hline 1 & Untrained & 5.23 & 220.75 & 398.85 \\
2 & Untrained & 5.30 & 223.06 & 454.62 \\
3 & Untrained & 5.32 & 200.76 & 324.55 \\
4 & Untrained & 5.30 & 198.97 & 343.92 \\
5 & Untrained & 5.20 & 209.22 & 332.71 \\
& Mean (std) & & $210.55 \pm 11.09$ & $370.93 \pm 55.06$ \\
\hline
\end{tabular}

Table 3. Data for the trained group at effort activity for 20 minutes

\begin{tabular}{crccc}
\hline Mouse & Status & RPM & Power average (IP) & Power max $($ MP) \\
\hline 1 & Trained & 5.24 & 220.94 & 202.02 \\
2 & Trained & 5.26 & 187.13 & 34.84 \\
3 & Trained & 5.29 & 210.43 & 291.41 \\
4 & Trained & 5.27 & 187.53 & 354.35 \\
5 & Trained & 5.29 & $201.61 \pm 14.66$ & 273.55 \\
& Mean $($ std) & & $325.97 \pm 41.03$ \\
\hline
\end{tabular}

Table 4. Data for the untrained (control) group at effort activity for 20 minutes

\begin{tabular}{ccccc}
\hline Mouse & Status & RPM & Power average (IP) & Power max (MP) \\
\hline 1 & Untrained & 5.25 & 230.94 & 382.84 \\
2 & Untrained & 5.26 & 222.02 & 360.70 \\
3 & Untrained & 5.25 & 199.13 & 315.41 \\
4 & Untrained & 5.27 & 231.43 & 361.35 \\
5 & Untrained & 5.22 & 203.53 & 295.55 \\
& Mean (std) & & $217.41 \pm 15.23$ & $343.17 \pm 36.23$ \\
\hline
\end{tabular}

$\mathrm{h}$ after the first one), the same effort was forced, but for a duration of 20 minutes for both groups.

All studies were performed on a specially designed commercial effort wheel (IntellioBio) connected to a computer. Data was collected using dedicated software. For both experimental trials, the speed used was $5 \mathrm{rpm}$, controlled by the computer. All values were expressed in average instantaneous power and maximum power and collected at 1 second intervals. Data was statistically analysed using ANOVA.

\section{Results}

No significant weight difference was found between the two mice groups, hence the influence of this parameter was excluded from further statistical interpretation. The average instantaneous power and maximum power at the 5 minutes experimental trial are shown in Tables 1 and 2 respectively. The average instantaneous power and maximum power at the 20 minutes experimental trial are shown in Tables 3 and 4 .

The instantaneous power developed by the effort wheel at 5 minutes was significantly lower $(\mathrm{p}<0.05)$ in the control group (IP $=210.55)$ than for the trained mice $(I P=201.53)$. Same results $(p<0.05)$ were obtained for the maximum power $(\mathrm{MP}=370.93$ for untrained mice; $\mathrm{MP}=346.80$ in trained mice). Interestingly, when the effort was prolonged, for the trained mice there was almost no difference between the average IP at 5 and 20 minutes of activity (IP $=201.53$ vs IP $=201.61$ ) nor between the maximum power obtained $(\mathrm{MP}=370.93$ vs $\mathrm{MP}=343.17)$. Nevertheless, the differences between the two groups remained statistically significant for both evaluated parameters $(\mathrm{p}<0.05)$.

\section{Discussion}

Voluntary wheel running is one of the most commonly studied behavioral activities in laboratory animals, with a history of more than 100 years (Sherwin, 1998). It has been used to evaluate many aspects, including the effect of parasitism, on a wide range of hosts, from rodents (Smith, 1978) to cockroaches (Moore, 1983) and of parasites, including the zoonotic Toxoplasma gondii (Hay et al., 1985).

The complex pathogenetic effect of the Trichinella larvae on the muscular tissue have been reviewed by Stewart (1995) as well as Bruschi and Chiumiento (2011). Several methods have been used to date to assess the behavioral changes in animals infected with Trichinella. Poirier et al. (1995) investigated the effect of two different species of Trichinella (T. nativa and $T$. 
pseudospiralis) on the diel locomotory activity of Peromyscus maniculatus. The methodology for evaluating the locomotory activity included an external infrared photorelay unit. Rau (1983) demonstrated that the ambulatory and exploratory activity of mice infected with $T$. spiralis decreased significantly as the infection progressed. The evaluation was done by open-field visual observation. Same experimental design was used one year later (Rau, 1984) for mice infected with T. pseudospiralis. Rau and Putter (1984) evaluated the running responses of CD-1 mice infected with T. spiralis and found an increased latency of the wall-seeking response and a decline in running speed and the distance travelled to the first stop.

However, none of these methods listed above have used an induced effort. They rather evaluated the voluntary, spontaneous or natural movement. In the opinion of the authors of the current study, the experimental model for the effort wheel developed herein allows the evaluation of resistance to a more intense effort, simulating the natural predator-escape behavior.

\section{Conclusions}

A marked difference between the two groups was noted, demonstrating the utility of using previously trained mice in experiments. As the differences between the values obtained at different exposure times were not significant for the trained mice, it was established that a duration of 5 minutes is sufficient for an experiment. Furthermore, the current results indicated that the present model can be a useful tool for evaluating the influence of Trichinella spp. on the muscular activity of experimentally infected mice in comparison to healthy ones from the same category.

\section{References}

Bruschi F, Chiumiento L (2011). Trichinella inflammatory myopathy: host or parasite strategy? Parasit Vectors 4:42.

Despommier DD (1983). Biology. In: Campbell W (Ed). Trichinella and Trichinellosis, Springer pp 75-152.

Hay J, Aitken PP, Arnott MA (1985). The influence of congenital Toxoplasma infection on the spontaneous running activity of mice. Zeitschriff für Parasitenkunde 71(4):459-462.

Moore J (1983). Altered behavior in cockroaches (Periplaneta americana) infected with an archiacanthocephalan, Moniliformis moniliformis. The Journal of Parasitology 69:11741776.

Moore J (2002). Parasites and the behavior of animals. Oxford University Press.

Novak CM, Burghardt PR, Levine JA (2012). The use of a running wheel to measure activity in rodents: relationship to energy balance, general activity, and reward. Neuroscience \& Biobehavioral Reviews 36(3):1001-1014.

Poirier SR, Rau ME, Wang X (1995). Diel locomotory activity of deer mice (Peromyscus maniculatus) infected with Trichinella nativa or Trichinella pseudospiralis. Canadian Journal of Zoology 73(7):13231334.

Rau ME (1983). The open-field behaviour of mice infected with Trichinellaspiralis. Parasitology 86:311-318.

Rau ME (1984). The open-field behaviour of mice infected with
Trichinella psendospiralis. Parasitology 88:415-419.

Rau ME, Putter L (1984). Running responses of Trichinella spiralisinfected CD-1 mice. Parasitology 89:579-584.

Sherwin CM (1998). Voluntary wheel running: a review and novel interpretation. Animal Behaviour 56:11-27.

Smith DH (1978). Effects of bot fly (Cuterebra) parasitism on activity patterns of Peromyscus maniculatus in the laboratory. Journal of Wildlife Diseases 14(1):28-39.

Stewart GL (1995). Myopathogenesis and myoredifferentiation in trichinosis. Basic and Applied Myology 5:213-222. 